\title{
Researches regarding the use of non-conventional actuators
}

\author{
I.C. Duțu1 ${ }^{1}$, T.Axinte ${ }^{2}$, E. Maican ${ }^{1}$, C. Frățilă ${ }^{2}$, R.G. Damian², E. Curcă², \\ V. Badanau ${ }^{2}$ \\ ${ }^{1}$ Politehnica University of Bucharest, Romania \\ ${ }^{2}$ Research and Innovation Center for Navy, Constanța, Romania \\ Corresponding author: T. Axinte, tibi_axinte@yahoo.com
}

\begin{abstract}
The purpose of this article is to present relevant concepts about the study of electropneumatic circuits using fluidic muscle actuators. The fluidic muscle is a type of pneumatic actuator having an extensive history of technical applications in the biomechanical field since the 1955. After Introduction, the authors study two pneumatic circuits. In fact, the first pneumatic circuit in this paper has only one actuator (fluidic muscle 1-1), but the second pneumatic circuit has two actuators (fluidic muscles 2-1 and 2-2. Further on, the authors present two electropneumatic schematics, a simple electro-pneumatic circuit and another electro-pneumatic circuit with PLC (Programmable Logic Controller). This type of actuator is used in robotics, material handling, motion control, industrial field and other applications. The pneumatic and electropneumatic circuits given in this paper are made using FluidSim software from Festo. In this case, the fluidic muscles are only non-conventional actuators. However, in pneumatic installations as well as in electro-pneumatic installations, the non-conventional actuators have the following advantages: strength, compactness, reliability, low price, ease of assembly or disassembly from their circuits, etc. Of course, in practice are many types of fluidic muscles, which are used in electro-pneumatic installations.
\end{abstract}

Keywords. Fluidic muscles, pneumatic, circuit, systems, FluidSim.

\section{Introduction}

A fluidic muscle is a tensile actuator which mimics the natural movement of a muscle. This type of actuator consist of a contractible tubing fitted with appropriate connectors.

The inventor, Richard Gaylord, discovered the fluidic muscle in 1955. He described this type of actuator as ,an elongated expansible tubular means surrounded by a woven sheath forming an expansible chamber which contracts in length when expanded circumferentially", [1].

The applications of fluidic muscles are:

- pneumatic springs;

- single-acting pneumatic actuators.

The contractible tubing is formed by a pressure-tight rubber hose, sheathed in high-strength fibers creating a rhomboidal pattern having a three-dimensional grid structure. The tubing stretches in circumferential direction when an internal pressure is applied, generating a tensile force and a contraction movement in the longitudinal direction of the muscle.

In fact, the tubing stretches in circumferential direction when an internal pressure is applied, generating a contraction movement and a tensile force in the longitudinal direction of the muscle.

However, the usable tensile force has its maximum value at the start of the contraction process, after that it decreases to zero in an almost linear manner as a function of stroke.

The fluidic muscles can be connected in the pneumatic installations by using:

- press-fitted connections;

- screwed connections. 
When the fluidic muscle is supplied with compressed air, it will contract in length, expanding radially, resulting a linear motion, as shown in figure 1, [2].

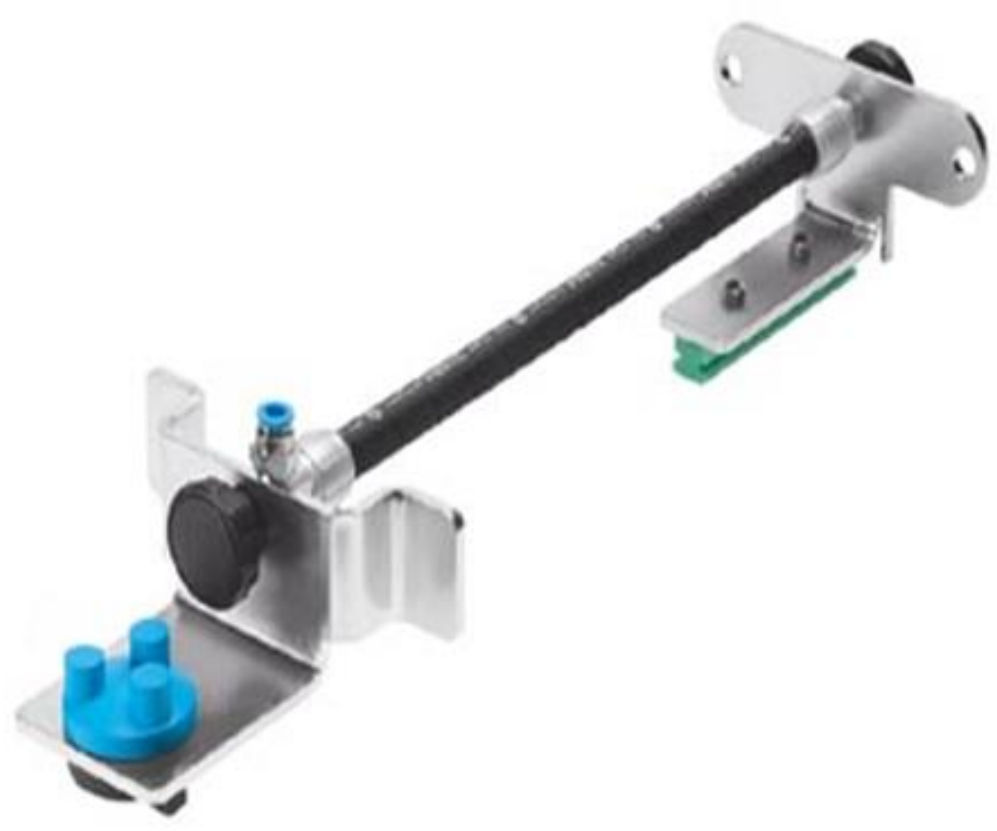

Figure 1. Fluidic muscle from Festo.

In practice, there are used various types of fluidic muscles, as shown in table 1. The most commonly used constructive types of fluidic muscles are: Kukolj muscle, McKibben muscle, Yarlott muscle, "rubbertuator" and pneumatic artificial muscle (PAM) from Festo, [3].

Table 1. Various types of fluidic muscles.

Constructive type name
Kukolj
McKibben of the fluidic muscle
"Rubbertuator"

A new type of pneumatic actuator is the bionic muscle. It consists of a contractile tube formed of a rubber diaphragm filled with strong, heat resistant fibers called aramid yarns, [4]. Considering the case when a fluidic muscle is filled with compressed air, it will increase in diameter and contract in length, providing sequences that closely approximate animal movements, as can be seen in figure 2 . 


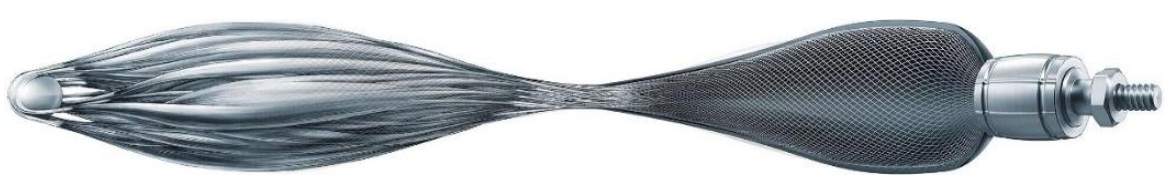

Figure 2. Rendition of the similarity between an actual human muscles and a fluidic muscle. A mathematical equation for the length of an inflated fluidic muscle can be written as:

$$
L=\sqrt{\frac{P b^{2}-4 \pi \cdot F \cdot n^{2}}{3 P}}
$$

In equation (1) there are given:

- $\quad P$, absolute pressure of the fluid;

- $b$, braided length of the fluidic muscle;

- $F$, force;

- $n$, number of turns of the thread.

The formula of the contraction ratio (relative contraction) of the fluidic muscle is given by:

$$
\varepsilon=\frac{L_{n}-L}{L_{n}} \cdot 100 \%
$$

In equation (2) are given:

- $L_{n}$, nominal length of the fluidic muscle;

- $L$, actual length of the fluidic muscle.

However, when calculating the force along the longitudinal axis, it can be neglected the deformation of membrane material and the low inertial muscle properties, [5]. In this case, the generated force $F$ can be written as:

$$
F=-p \frac{d V}{d l}
$$

where:

- $\quad p$ - the gauge pressure inside the muscle;

- $d V$ - enclosed muscle volume changes;

- $d l$ - actuator length changes.

\section{Pneumatic circuits using fluidic muscles}

The following pneumatic circuits presented in the paper are operating only when the $3 / 2$ - way air directional valve will be given a manual command, using $S 1$, [6].

Table 2. Devices in the first pneumatic circuit.

\begin{tabular}{lc}
\hline Description & Number of components \\
\hline Compressed air supply & 1 \\
Start-up valve & 1 \\
$3 / 2$ - way directional valve & 1 \\
Throttle check valve & 1 \\
Fluidic muscle & 1
\end{tabular}


The first pneumatic circuit that the authors have studied has one fluidic muscle 1-1, as shown in figure 3.

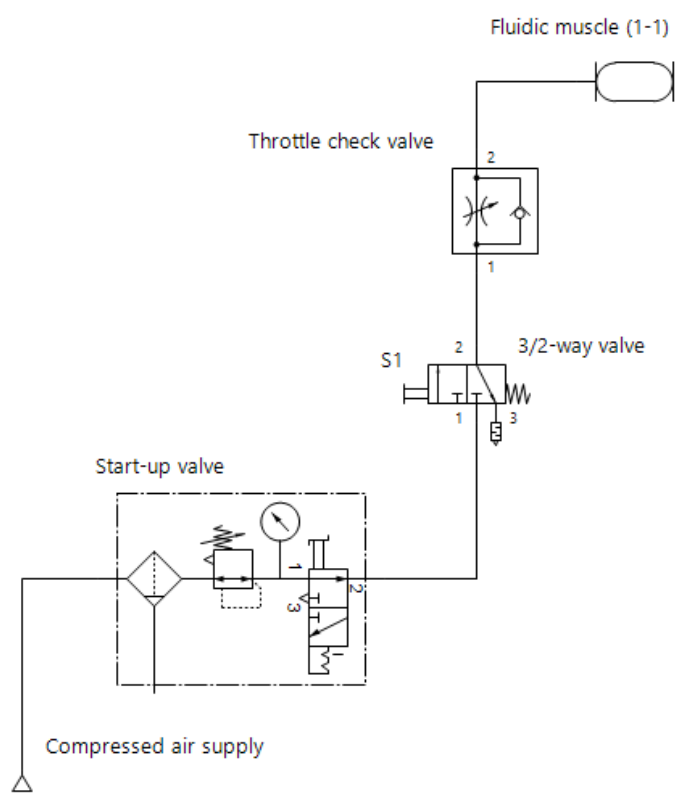

Figure 3. First pneumatic circuit using one fluidic muscle.

The first pneumatic circuit operates if the operator presses $S 1$ button of the $3 / 2$ way directional valve, as shown in figure 4 .

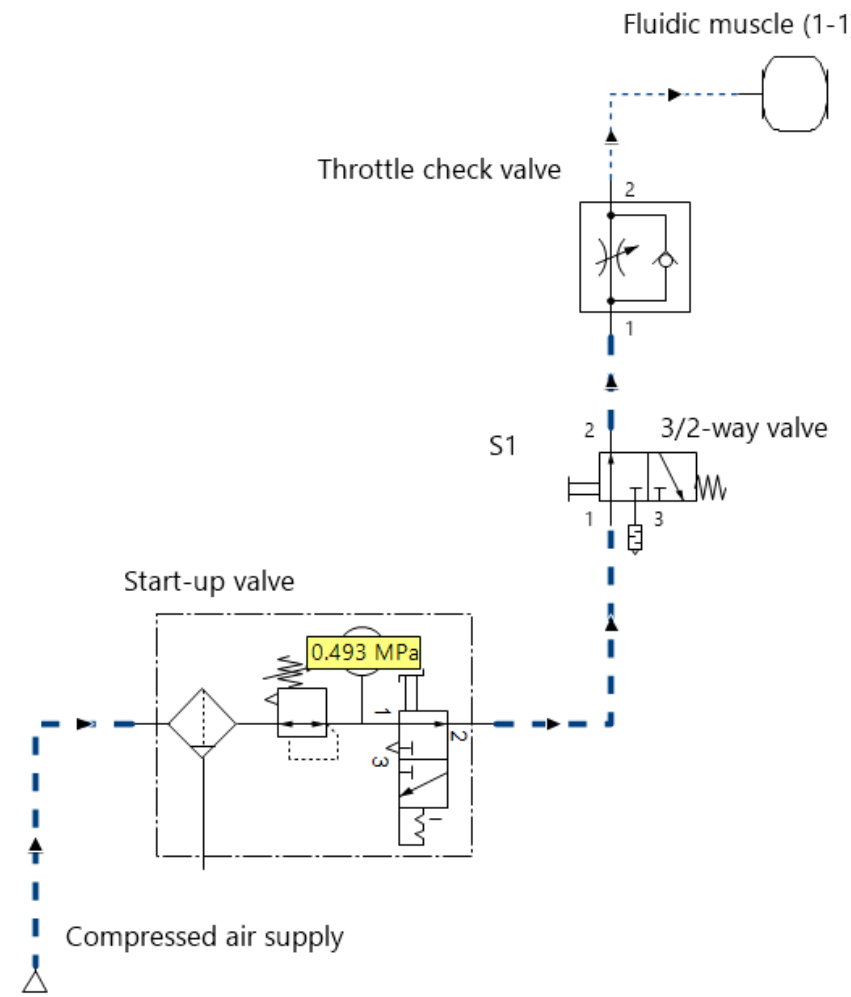

Figure 4. Simulation of the first pneumatic circuit.

The diagrams given in figure 5 show variation of the following functional parameters of the fluidic muscle:

- contraction $-h[\%]$;

- position $-x[\mathrm{~mm}]$;

- velocity $-v[\mathrm{~m} / \mathrm{s}]$;

- acceleration $-a\left[\mathrm{~m} / \mathrm{s}^{2}\right]$. 


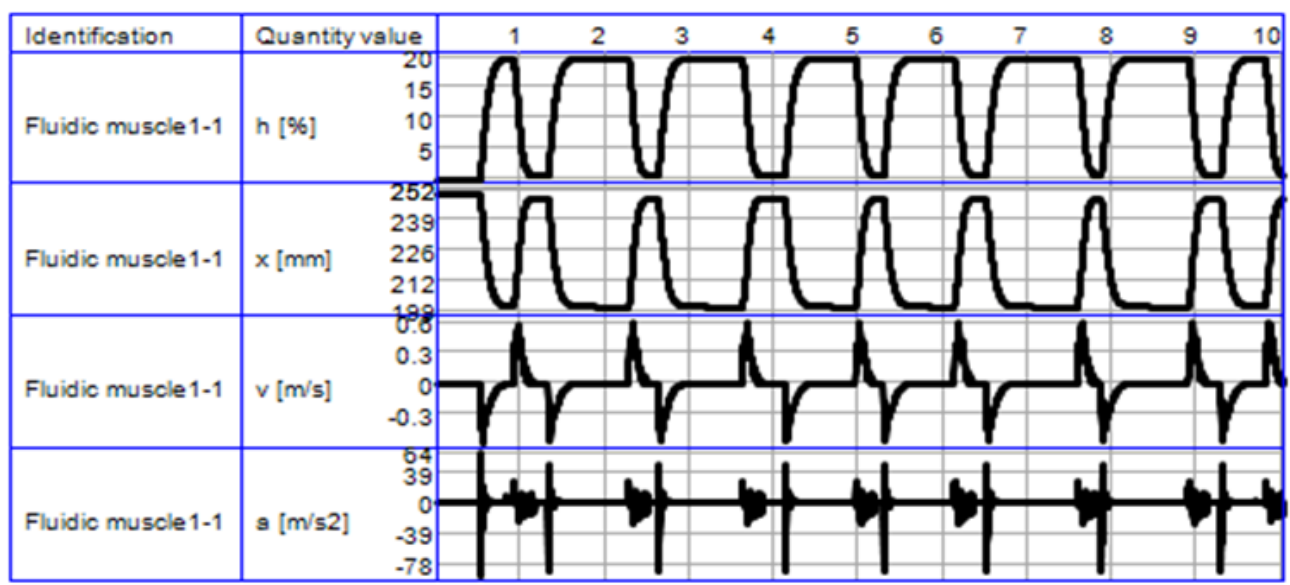

Figure 5. Diagrams of functional parameters variations of the fluidic muscle (1-1).

In the table below there are given the component pneumatic devices used in the second circuit.

Table 3. Devices in the second pneumatic circuit.

\begin{tabular}{lc}
\hline Description & Number of components \\
\hline Compressed air supply & 1 \\
Air filter & 1 \\
$4 / 2$ - way directional valve & 1 \\
Throttle check valve $1 \& 2$ & 2 \\
Fluidic muscles $(2-1) \&(2-2)$ & 2
\end{tabular}

The second pneumatic circuits that the authors have studied uses two fluidic muscles, (2-1) and (22 ), as can be seen in figure 6 .

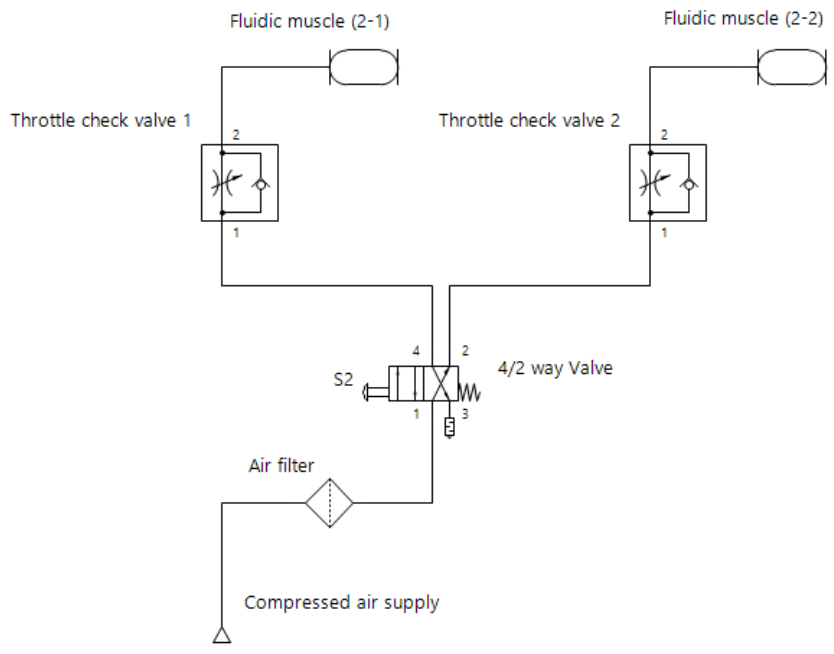

Figure 6. Second pneumatic circuit using two fluidic muscles.

In figure $7 \mathrm{a}$ and $7 \mathrm{~b}$ the authors show two functional states of the circuit given above in figure 6 , during the simulation in FluidSim. In the initial state, at circuit power-on, it can be seen that the fluidic muscle 2-2 will begin to gradually expand in its peripheral direction, using the air flow through the throttle check valve 2 . In the case the operator presses and holds button $S 2$, the fluidic muscle 2-1 will begin to expand while $2-1$ will deflate. 


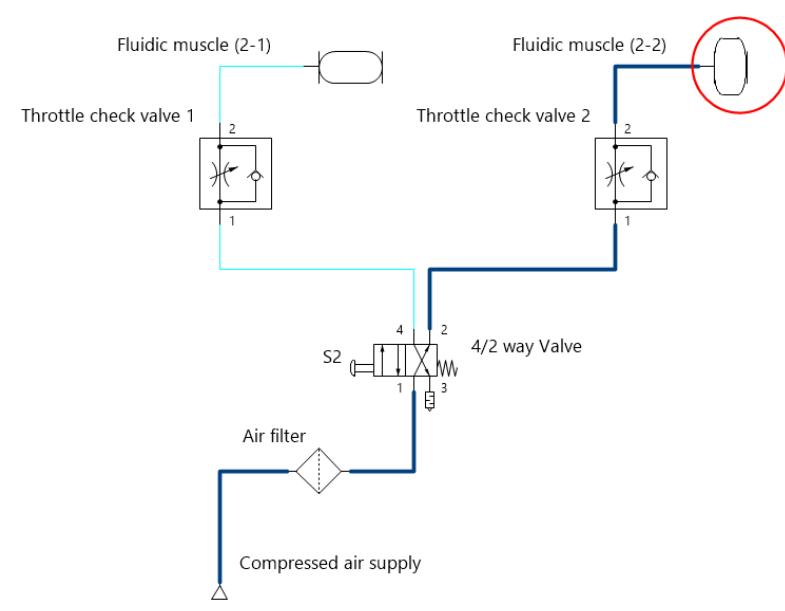

a. initial state of the circuit (power-on);

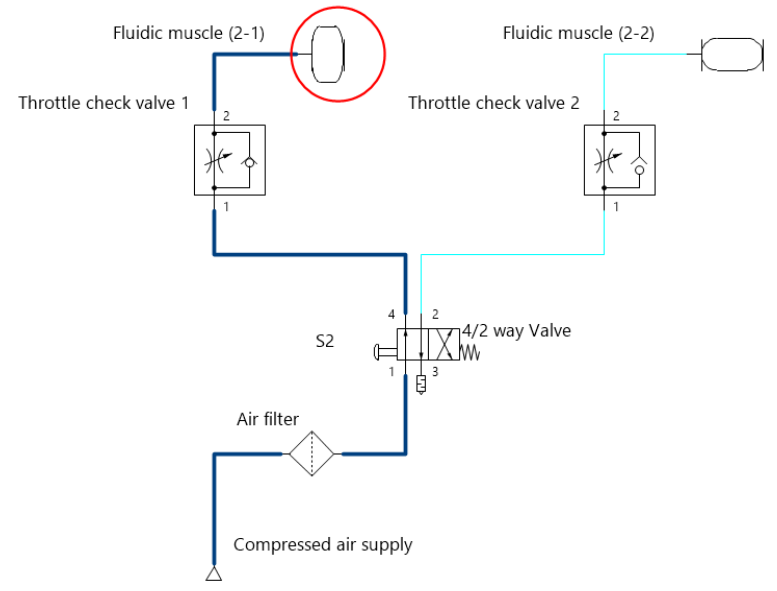

b. state of the circuit with $S 2$ pressed;

Figure 7. Simulation of the second pneumatic circuit.

\section{Electro-pneumatic circuits using fluidic muscles}

Electro-pneumatic circuits containing fluidic muscles are more complex than the pneumatic ones using as an actuator the same fluidic muscle, [7].

When comparing with pneumatic circuits, the electro-pneumatic circuits essentially demonstrate the following advantages:

- simple and rapid modification of open and closed control strategies using programmable logic controllers (PLCs);

- easy exchange of information between several control systems;

- less effort and expense for planning and commissioning;

- less time required for installation, [8].
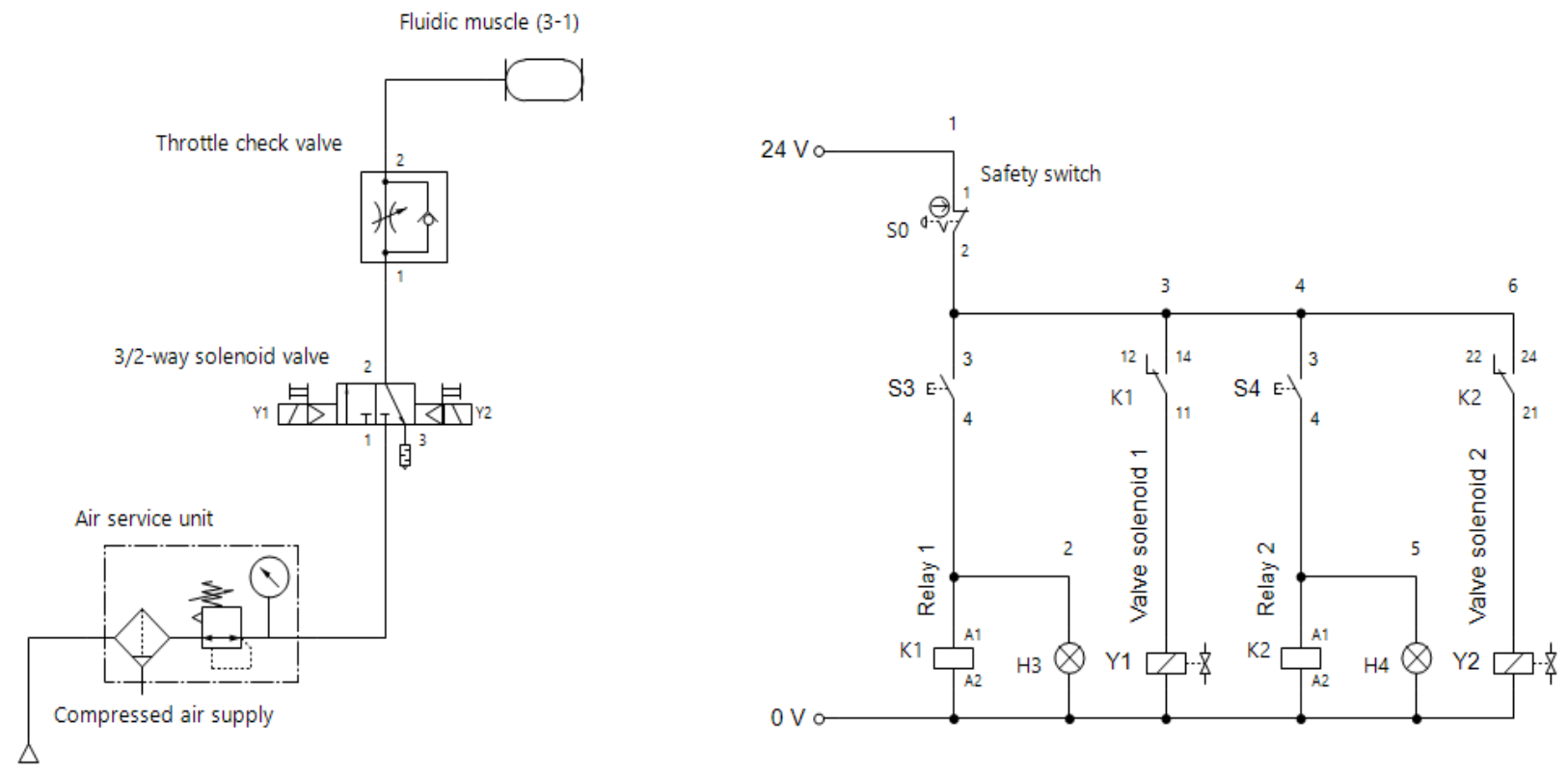

Figure 8. First electro-pneumatic circuit.

The first electro-pneumatic circuit, as shown in figure 8, comprises a few basic electrical and pneumatic devices, given in the table below. It must be noted that the authors used a latched 3/2-way solenoid valve, having a "memory" effect on the commands issued by the operator. 
Table 4. Devices in the first electro-pneumatic circuit.

\begin{tabular}{lc}
\hline Description & Number of components \\
\hline Fluidic muscle $(3-1)$ & 1 \\
Throttle check valve & 1 \\
$3 / 2$ - way solenoid valve & 1 \\
Valve solenoids, $Y 1 \& Y 2$ & 2 \\
Air service unit & 1 \\
Command relays, $K 1 \& K 2$ & 2 \\
Signal lamps, $H 1 \& H 2$ & 2 \\
$N O$ push-buttons $S 3 \& S 4$ & 2 \\
$N C$ safety switch, $S O$ & 1
\end{tabular}

In order to operate the electro-pneumatic circuit shown in figure 8, having one fluidic muscle (3-1), the operator has to press the $S 3$ button. Then, the fluidic muscle will begin to gradually expand in its peripheral direction, as shown in figure 9.
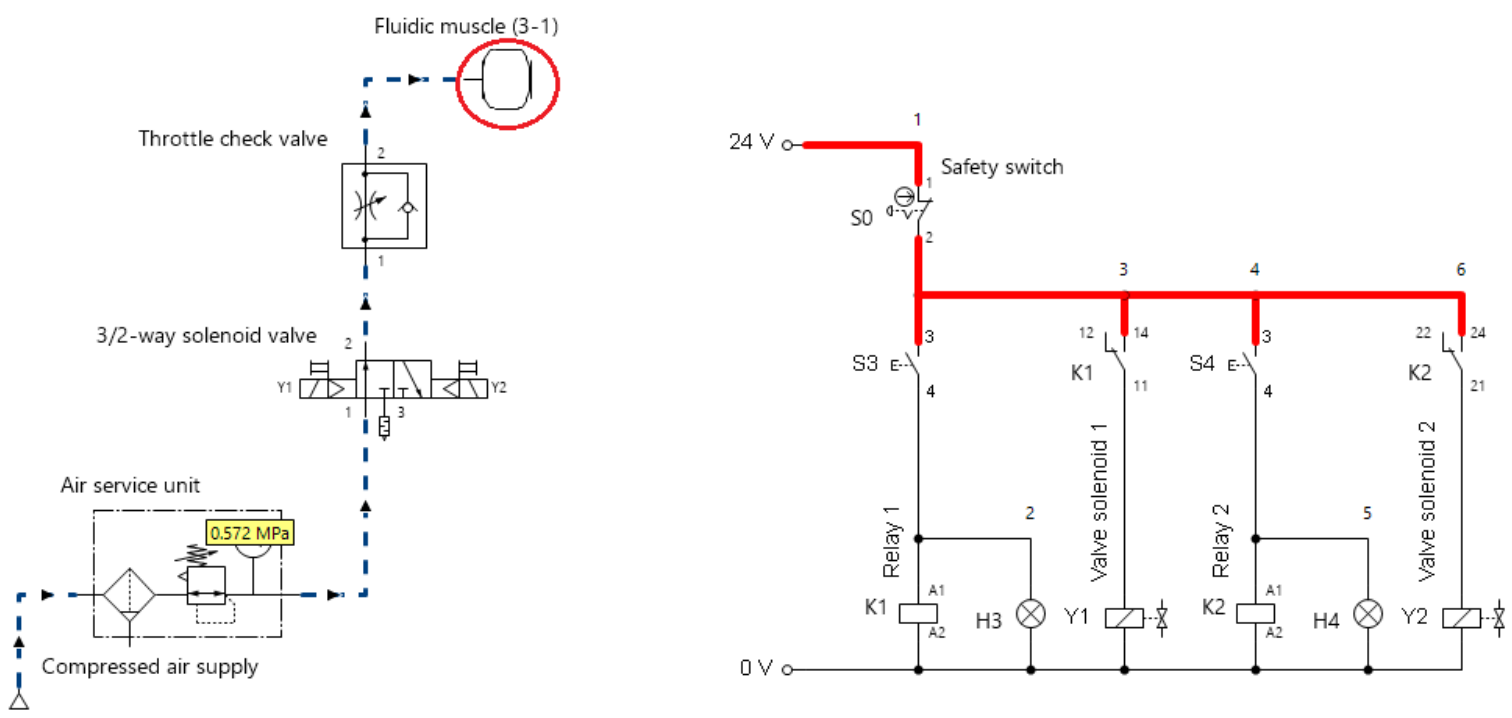

Figure 9. Contraction process simulation of fluidic muscle (3-1).

For deflating the fluidic muscle (3-1), in order to return to its initial state, the operator has to press button $S 4$, after the button $S 3$ has been released. Now, the fluidic muscle (3-1) will start to deflate, as can be seen in figure 10 .

Here, also, the authors used a latched 3/2-way solenoid valve, in order to benefit from the "memory" effect of this device on the commands issued by the operator. Another solution is using a non-latching valve and a simple electric circuit that stores the commands issued by the $S 3$ or $S 4$ buttons. In this case the circuit must be provided with a supplementary function that disables the operator's possibility to press $S 3$ and $S 4$ at the same time. This alternative electric circuit can use $K 1$ and $K 2$ relays to obtain the latching effect and disabling function, [9].

As can be seen in figure $9, K 1$ and $K 2$ relays have changeover contacts, but it can be used another constructive type of electromechanical relays having normally-open $(N O)$ and normally-closed $(N C)$ pairs of contacts. Eventually, it can be used a supplementary lamp to indicate circuit's power-on state. 

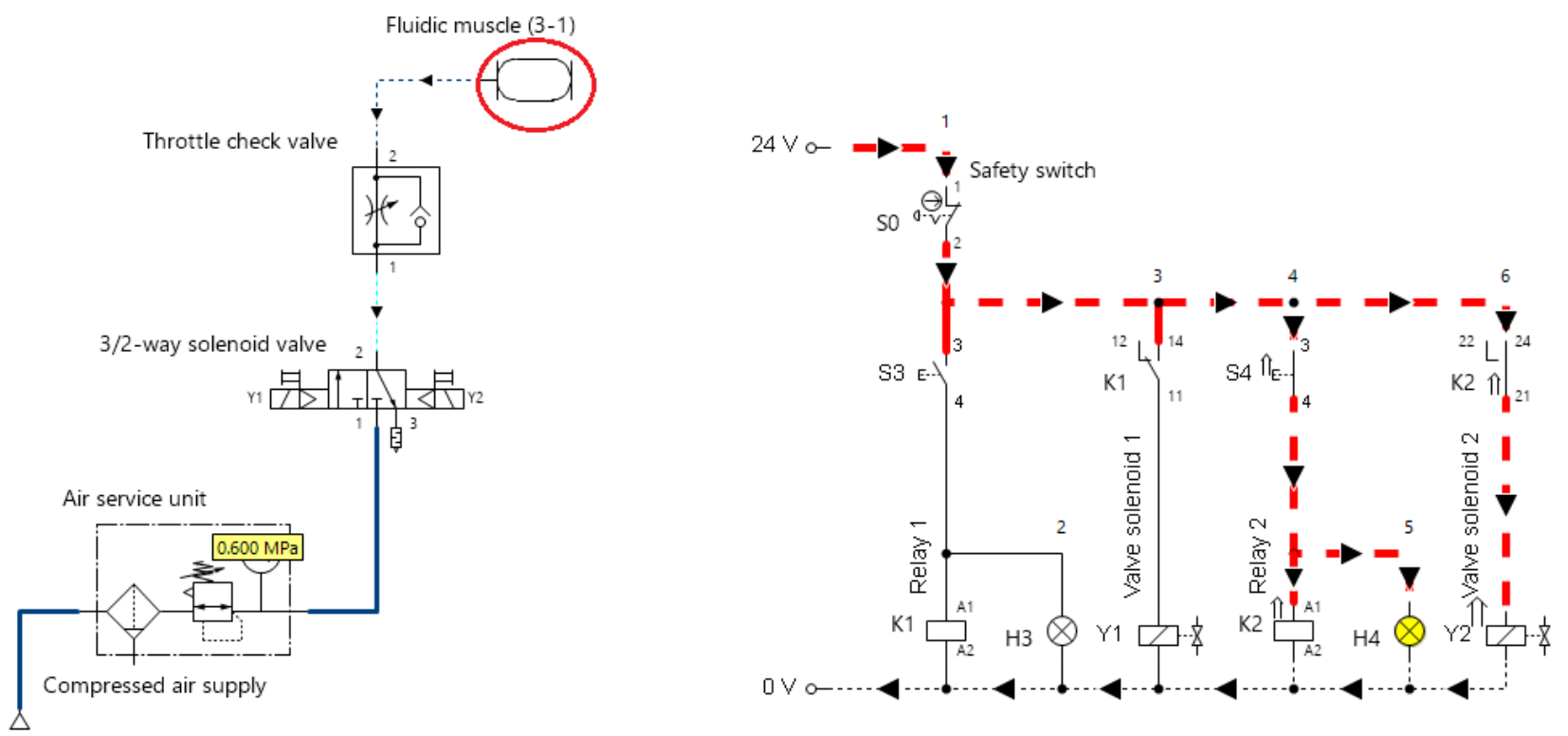

Figure 10. Deflating process simulation of fluidic muscle (3-1).

Another further improvement for the circuits presented above is the use of a programmable logic controller $(P L C)$, providing much more flexibility in controlling the fluidic muscle, as can be seen in figure 11 .
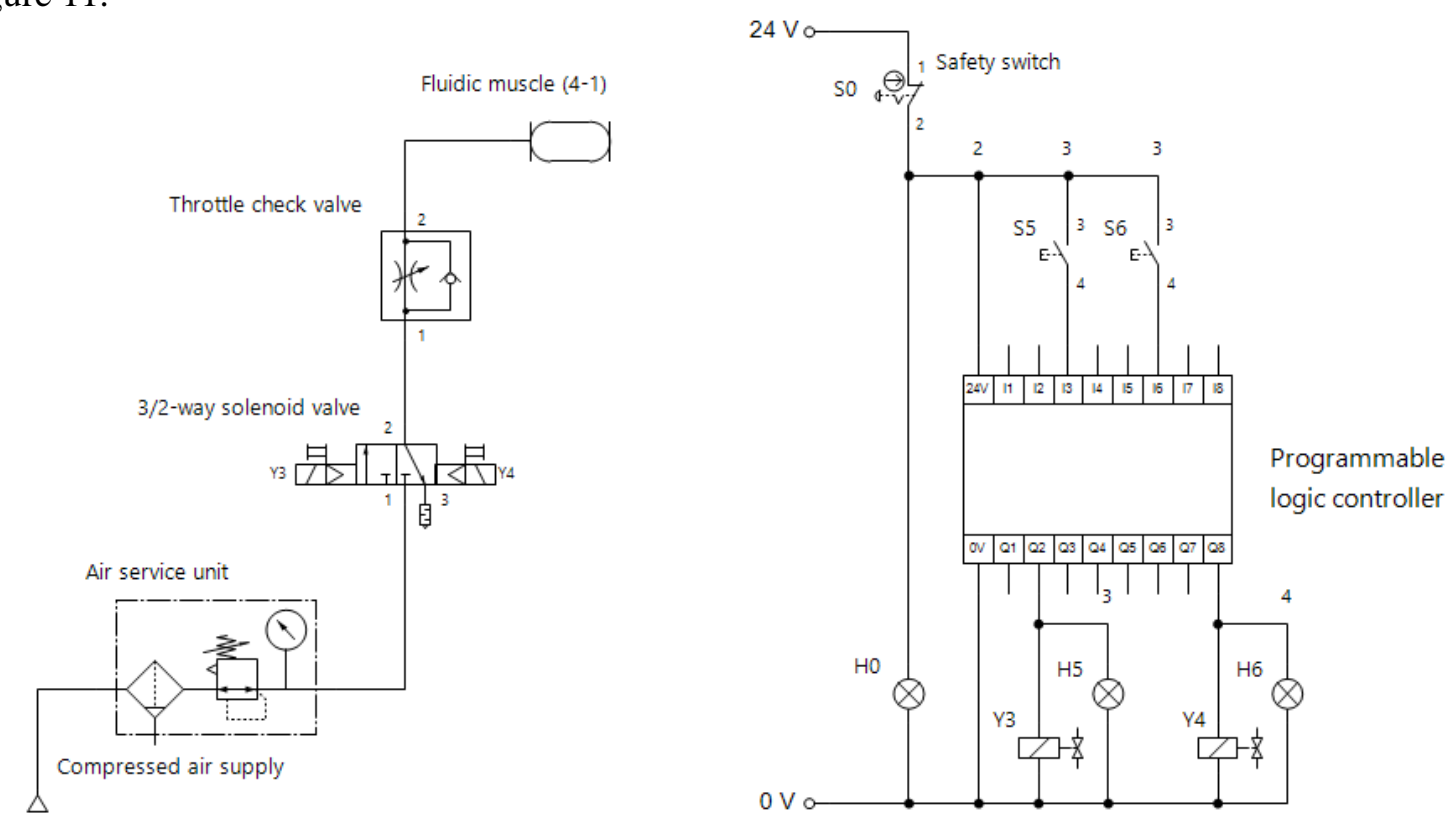

Figure 11. Second electro-pneumatic circuit using a programmable logic controller.

The programmable logic controller, used in the circuit given above, uses one 8-bit digital input port for interfacing $S 5$ and $S 6$ command buttons and one 8-bit digital output port for issuing commands to electric circuits $Y 3 / H 5$ and $Y 4 / H 6$. The internal programmable logic can be configured in such way that it can provide latching and conditional enable or disable functions, substituting external hardware circuits. The digital output port of the controller, in the configuration that the authors used, have relays for each single output that replace $K 1$ and $K 2$ electromechanical relays from figure 8 . The authors used $I 3$ and $I 6$ input bits and $Q 2$ and $Q 8$ for output bits, all using $24 \mathrm{~V}$ DC voltage level, but having different current ratings per port type.

In the table below, there are given some important devices used in the second electro-pneumatic circuit, [10]. 
Table 5. Devices in the first electro-pneumatic circuit.

\begin{tabular}{lc}
\hline Description & Number of components \\
\hline Fluidic muscle (4-1) & 1 \\
Throttle check valve & 1 \\
$3 / 2$ - way solenoid valve & 1 \\
Valve solenoids, $Y 3 \& Y 4$ & 2 \\
Air service unit & 1 \\
Signal lamp, HO, $H 5 \& H 6$ & 3 \\
NO push-buttons $S 5 \& S 6$ & 2 \\
$N C$ safety switch, $S O$ & 1 \\
Programmable logic controller & 1
\end{tabular}

In the next two figures there are shown simulations for the contraction and deflating proceses of the fluidic muscle (4-1), based on the circuit given in figure 11 .
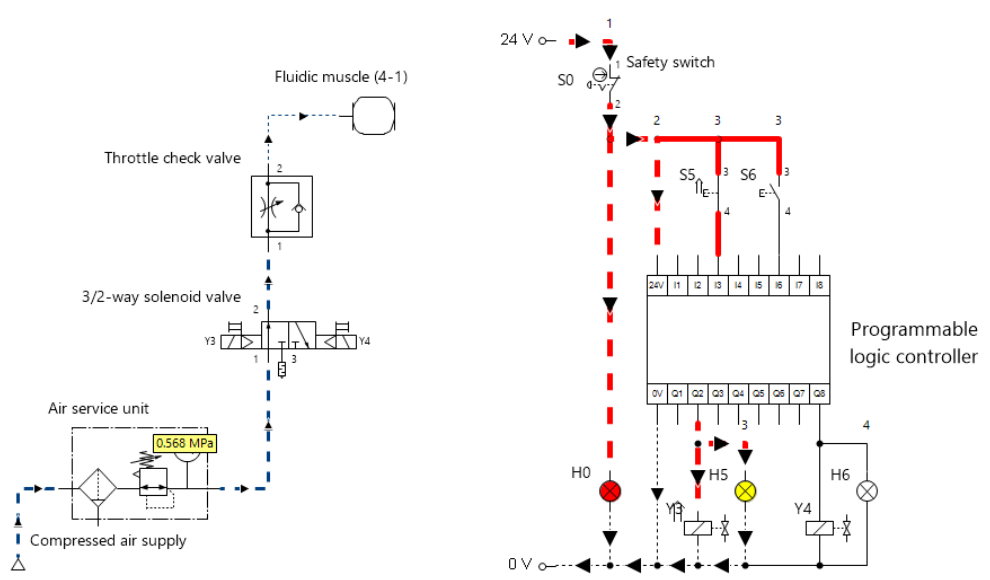

Figure 12. Contraction process simulation of fluidic muscle (4-1).

As can be seen in both figures 12 and 13, the programmable logic controller has the role of issuing commands to the $3 / 2$-way valve, having the possibility to be reprogrammed in case of working algorithm changes, circuit updates or using another fluidic muscle with new parameters.
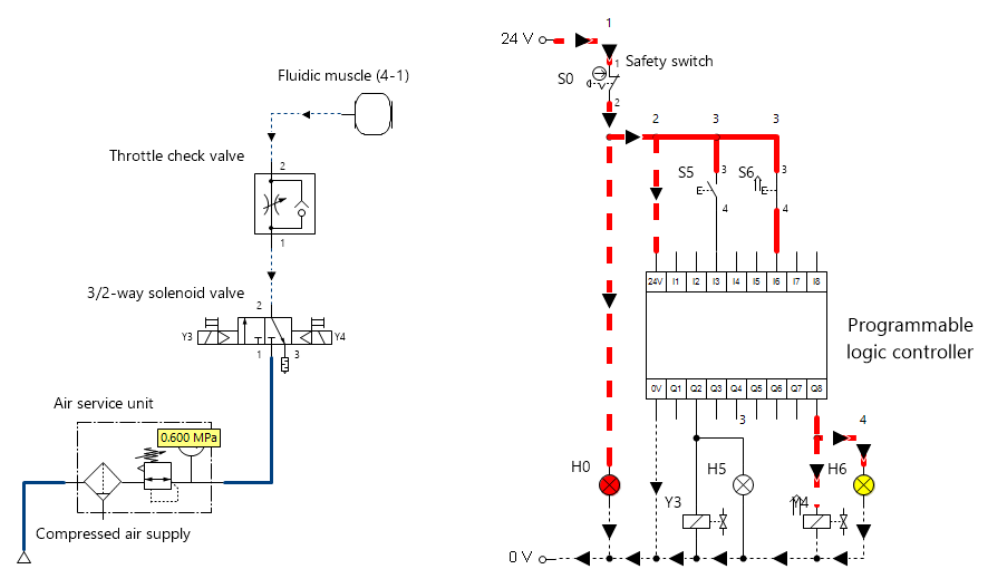

Figure 13. Deflating process simulation of fluidic muscle (4-1). 


\section{Conclusions}

Taking into consideration the presented above electro-pneumatic circuits using a fluidic muscle, it can be seen that these can offer several advantages over traditional electro-pneumatic systems using another type of actuators, such as semi-rotary, air motor, etc. Some important advantages to be noted are: lower cost, higher power to weight ratio, less weight, no restriction regarding working in dusty environments, etc.

Therefore, given the superior strength, it may seem that fluidic muscles may replace, in particular applications, pneumatic cylinders from electro-pneumatic installations.

However, this unconventional type of actuator offer some unique features that must be explored by users in a wide variety of fields such as robotics, production equipment, artificial muscles, biomechanics based algorithms, material handling, etc.

The users and researchers should be encouraged to take into account the fluidic muscle when searching for a suitable fluid actuator that can be easily integrated in the electro-pneumatic installations using programmable logic controller (PLC).

The non-conventional actuators are often used in areas of extreme temperatures due to the safety of using than hazardous electricity or chemicals.

In conclusion, these non-conventional actuators are very simple devices providing a low-cost alternative.

\section{Acknowledgement}

This research was supported by the Executive Unit for Financing Higher Education, Research, Development and Innovation, UEFISCDI, under the PNCDI III - Programme 2, sub-programme 2.1, as follows partially from:

- submission code PN-III-P2-2.1-PTE-2019-0446, funding contract no. 53PTE/23.09.2020, project title "Hydrophilic auto-chassis for high energy efficiency operation of interchangeable equipment intended for performing public utility work", acronym ASHEUP, research direction 3.Econano-technologies and advanced materials, subdomain Advanced materials;

- submission code PN-III-P2- 2.1-PED-2019-4123, funding contract no. 380PED/23.10.2020, project title "Multifunctional hexa-rotors tricopter for precision farming environment", acronym 4.0-MHRT, project domain 1.Bioeconomics, subdomain 1.1.

\section{References}

[1] S. LIGHTNER, R. LINCOLN: The Fluidic Muscle: A 'New' Development, Journal of Modern Engineering, vol. 2, no. 2, 2002.

[2] ***, https://www.festo-didactic.com/ro-ro/, last accessed on 10 October 2021.

[3] E. KELASIDI, G. ANDRIKOPOULOS, G. NIKOLAKOPULOS, S. MANESIS: A Survey on Pneumatic Muscle Actuators Modeling, Journal of Energy and Power Engineering, 2012.

[4] ***, http://www.asknature.org, last accessed on 5 October 2021.

[5] I. VENEVA, B. VANDERBORGHT: Propulsion System with Pneumatic Artificial Muscles for Powering Ankle-Foot Orthosis, Journal of Theoretical and Applied Mechanics, vol. 43, no. 4, pp. 3-16, doi: 10.2478/jtam-2013-0029, Sofia, Bulgaria, 2013.

[6] I. PANAITESCU, A. SCUPI, V. PANAITESCU, M. PANAITESCU: Efficient Use of the Pumps in the Wastewater Treatment Plant, CIEM, Politehnica ISSN 2067-0893, Bucharest, 2013.

[7] ***, http://www.iamtechnical.com, last accessed on 29 September 2021.

[8] B.C. BELEV: Information Capabilities of Integrated Bridge Systems, Journal of Navigation 57(01): pp. 145-151, doi: 10.1017/S0373463303002492, 2004.

[9] S. FABRIZIO, D. ACCOTO, G. CARPINO: Design and Characterization of a Compact Rotary Series Elastic Actuator for Knee Assistance During Overground Walking, International Conference on biomedical Robotics and Biomechatronics, doi: 10.1109/BioRob.2012.6290271, 2012.

[10] C.L. DUMITRACHE, D. DELEANU: Computational NX Fluid Structure Interaction (FSI) analysis on naval three way ball valve, Conference Series: Materials Science and Engineering, ModTech, doi: 10.1088/1757-899X/1182/1/012021, 2021. 